\title{
Electronic Structure of $\mathrm{PrCo}_{2} \mathrm{Ge}_{2}$
}

\author{
M. Diviš, J. Vejpravová, J. Rusz, J. ProkleŠka \\ AND V. SECHOVSKÝ \\ Department of Condensed Matter Physics, Charles University \\ Faculty of Mathematics and Physics \\ Ke Karlovu 5, 12116 Prague 2, Czech Republic
}

\begin{abstract}
The intermetallic compound $\mathrm{PrCo}_{2} \mathrm{Ge}_{2}$ exhibits very interesting physical properties: antiferromagnetism below $T_{\mathrm{N}}=28 \mathrm{~K}$, an order to order transition at $T_{\mathrm{R}}=8 \mathrm{~K}$, a rich magnetic phase diagram, and huge magnetocrystalline anisotropy as observed by recent experiments on a single crystal in a magnetic field up to $14 \mathrm{~T}$. We performed first principles calculations based on the density functional theory. The localized $4 f^{2}$ configuration of $\mathrm{Pr}^{3+}$ was treated using the open core approximation. The electronic densities of states were obtained and the position of the Fermi level was found. The hybridization between the Co- $3 d, \operatorname{Pr}-5 d$ and Ge- $4 p$ states is discussed. The calculated magnetic moment of $\mathrm{Co}$ is found to be quite small $\left(m_{\mathrm{Co}}<0.1 \mu_{\mathrm{B}}\right)$. The first principles calculations of the crystal field interaction were performed as well: the obtained crystal field parameters were used as the starting parameters for further refinement in comparison with experimental data of the magnetic susceptibility and specific heat. The crystal field splitting of the $4 f$ states was determined.
\end{abstract}

PACS numbers: 71.15.Mb, 71.70.Ch

\section{Introduction}

The family of the $\mathrm{RT}_{2} \mathrm{X}_{2}(\mathrm{R}=$ rare earth, $\mathrm{T}=$ transition metal, $\mathrm{X}=p$ element) intermetallic compounds is one of the largest among ternary systems. In these materials a large variety of physical phenomena has been observed, among others the dense Kondo behavior, mixed valence phenomena, exotic magnetic ground states sometimes coexisting with superconductivity, which are always accompanied by very strong uniaxial magnetic anisotropy.

Majority of these compounds crystallize in the tetragonal body-centered structure (space group $I 4 / \mathrm{mmm}$ ), which can be described as layers of atoms of the same kind stacked along the tetragonal axis in the sequence $\mathrm{R}-\mathrm{T}-\mathrm{X}-\mathrm{T}$. The physical, especially magnetic properties of the $\mathrm{RT}_{2} \mathrm{X}_{2}$ compounds reflect the layered nature of the crystal structure. 
The early X-ray diffraction of $\mathrm{PrCo}_{2} \mathrm{Ge}_{2}$ polycrystalline sample shows [1] tetragonal crystal structure belonging to the space group $I 4 / \mathrm{mmm}$.

Subsequent neutron diffraction confirmed [2] the antiferromagnetic ordering at $27 \pm 1 \mathrm{~K}$ with propagation vector $(0,0,0.72)$.

In this work we present the first principles calculations of the electronic structure and crystal field using density functional theory. The comparison with our single crystal susceptibility and specific heat data is provided.

\section{Method of calculations, results, and discussion}

We used state-of-the art computational methods for the electronic structure calculations, namely the full-potential linearized augmented plane waves method (LAPW, WIEN2k code) [3]. The Kohn-Sham equations were solved within the local-spin-density approximation (LSDA) [3] and the generalized gradient approximation (GGA) [3]. The relativistic effects of the valence states were treated within the scalar relativistic approximation [3], core states were described using a four-component fully relativistic Dirac solver. The correlated $4 f$ states of Pr were treated in the open core approximation, and thus, the praseodymium $4 f$ states are characterized by an integer occupation number $4 f^{2}$. The number of APW, $k$-points, and the Fourier coefficients were carefully checked to ensure convergence of the calculations. The first principles crystal field calculations were performed using the method described in Divis et al. [4].

The calculated electronic density of states (DOS) of $\mathrm{PrCo}_{2} \mathrm{Ge}_{2}$ for the experimental lattice parameters in normal paramagnetic state is shown in Fig. 1a. The first region, from $-11.1 \mathrm{eV}$ to $-7.6 \mathrm{eV}$, consists mainly of the Ge- $4 s$ states, which are split off from the main valence band group (see Fig. 1d). There is a band gap from $-7.6 \mathrm{eV}$ to $-5.3 \mathrm{eV}$. The bottom of the main valence band group is situated at $-5.3 \mathrm{eV}$. This band group between $-5.3 \mathrm{eV}$ and $2 \mathrm{eV}$ represents mainly the cobalt $3 d$ states with the major part located below the Fermi level $\left(E_{\mathrm{F}}\right)$, forming a pronounced broad maximum in the DOS curve between $-5.3 \mathrm{eV}$ to $-0.55 \mathrm{eV}$, a quasigap situated below $E_{\mathrm{F}}$, and a secondary maximum just above $E_{\mathrm{F}}$. There is some hybridization between the Co-3d (see Fig. 1c), Pr-5d (see Fig. 1b) and Ge- $4 p$ (see Fig. 1d) states present between $-3 \mathrm{eV}$ and the $E_{\mathrm{F}}$ and a pronounced contribution originating from the interstitial region (see dashed line, Fig. 1d). The energy position of the localized $4 f^{2}(j=5 / 2)$ states is below $E_{\mathrm{F}}$.

$E_{\mathrm{F}}$ is situated at the end of the quasigap between the two pronounced maxima in the DOS. The orbital analysis of the DOS shows that mainly the Co- $3 d$, Pr- $5 d$, and Ge- $4 p$ states contribute to the total value of DOS at the $E_{\mathrm{F}}$. The value of the DOS at $E_{\mathrm{F}}$ is too small to cause the spontaneous magnetic polarization of the Co- $3 d$ states. The peculiar position of $E_{\mathrm{F}}$ corroborates the idea that the absence of the cobalt magnetic moment is due to the itinerant character of the Co-3d states connected with the position of $E_{\mathrm{F}}$ at the quasigap. The value of the DOS for $\mathrm{PrCo}_{2} \mathrm{Ge}_{2}$ and $\mathrm{LaCo}_{2} \mathrm{Ge}_{2}$ at the $E_{\mathrm{F}}$ corresponds to $\gamma=9.8 \mathrm{~mJ} /\left(\mathrm{mol} \mathrm{K}{ }^{2}\right)$ 


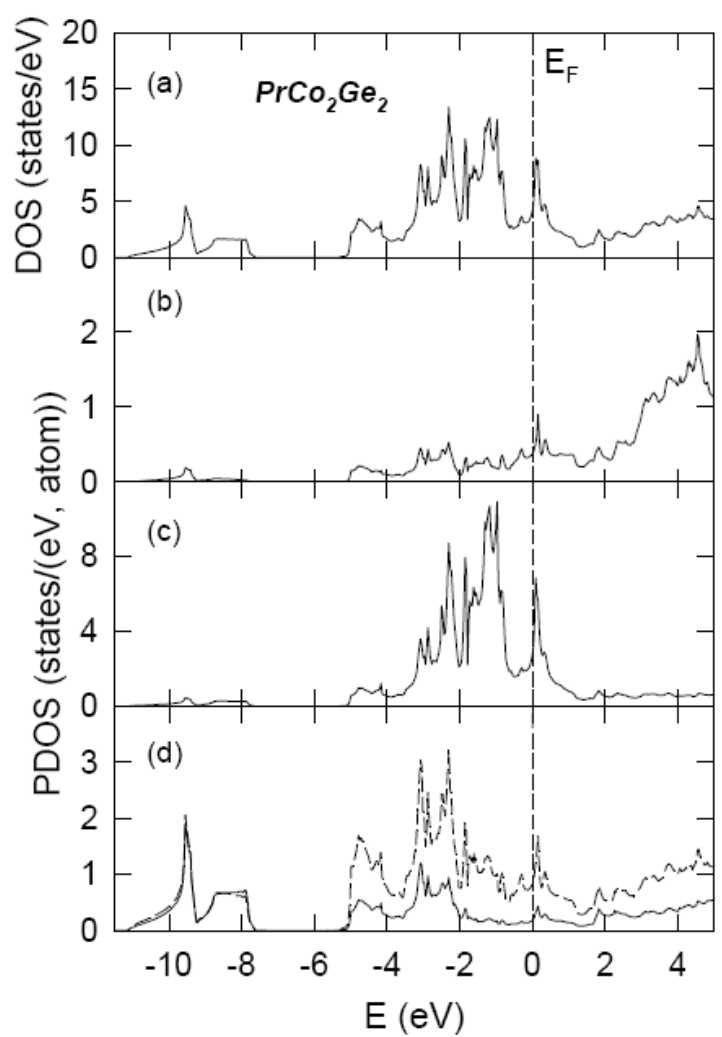

Fig. 1. The total DOS (a) and atom projected DOS (b), (c), (d) of $\mathrm{PrCo}_{2} \mathrm{Ge}_{2}$.

and $11.1 \mathrm{~mJ} /\left(\mathrm{mol} \mathrm{K}^{2}\right)$, respectively, which is lower than the $\gamma$ value obtained from the analysis of our specific heat data for $\mathrm{LaCo}_{2} \mathrm{Ge}_{2}$. This points to a rather low value of the mass enhancement coefficient $\lambda=0.48$ for $\mathrm{LaCo}_{2} \mathrm{Ge}_{2}$ which is related to a small electron-phonon interaction in the $\mathrm{RCo}_{2} \mathrm{Ge}_{2}$ compounds.

In the case of $\mathrm{LaCO}_{2} \mathrm{Ge}_{2}$ the minimization of the total energy with respect to volume provided $V / V_{0}=0.936$. This is the usual underestimation provided by the LSDA. We repeated the whole procedure using the GGA form of the exchange-correlation potential and found $V / V_{0}=1.004$, which demonstrates that the GGA calculations provide a much better agreement with the experimental equilibrium volume $V_{0}$. We did not minimize the force acting on the Ge atom since the calculated force was already quite small. The fact that the GGA provided a better ground state density also suggested to perform the first principles crystal field calculations using the GGA ground state density.

For the purpose of crystal field (CF) fitting the magnetization was measured in the range $2-600 \mathrm{~K}$ in several magnetic fields and the magnetic susceptibility in the paramagnetic region was taken as a slope of the $M(B)$ data at constant temperature. The CF Hamiltonian in the tetragonal symmetry depends on five 
CF parameters $A_{n}^{m}$. The first principles calculation of the CF parameters leads to the following values: $A_{2}^{0}=158 \mathrm{~K}, A_{4}^{0}=-13 \mathrm{~K}, A_{4}^{4}=600 \mathrm{~K}, A_{6}^{0}=-4.6 \mathrm{~K}$, and $A_{6}^{4}=61 \mathrm{~K}$ using the GGA form of the exchange-correlation potential. The second-order $\mathrm{CF}$ parameter $A_{2}^{0}$ has the correct sign, which determines the easy $c$-axis of $\mathrm{PrCo}_{2} \mathrm{Ge}_{2}$ in agreement with experiment and with the analysis of our susceptibility data. The parameter $A_{6}^{0}$ has the correct sign comparing to the CF parameters resulting from analysis of susceptibility. Since the analysis of our susceptibility data is not very sensitive to the values of off-diagonal CF parameters $A_{4}^{4}$ and $A_{6}^{4}$, there is also agreement between first principles calculation and the $\mathrm{CF}$ parameters obtained from the susceptibility.

We fitted the temperature dependence of the susceptibility to this model CF Hamiltonian by an explicit parameter space search and a simulated annealing procedure [5] and obtained the same results within both methods. The CF parameters are $A_{2}^{0}=317 \mathrm{~K}, A_{4}^{0}=49 \mathrm{~K}, A_{4}^{4}=719 \mathrm{~K}, A_{6}^{0}=-85 \mathrm{~K}$ and $A_{6}^{4}=681 \mathrm{~K}$ and a constant shift $35.1 \mathrm{~T} / \mu_{\mathrm{B}}$. This shift corresponds to a paramagnetic critical temperature $47.8 \mathrm{~K}$, in a reasonable agreement with experimental findings (taking into account the mean-field approximation). The agreement between the model calculations and experiment (susceptibility, specific heat) confirms a dominant role of crystal field effects and an important role of exchange interactions in the system.

\section{Acknowledgments}

This work is a part of the research plan MSM 0021620834 that is financed by the Ministry of Education of the Czech Republic. Financial support of the Grant Agency of Czech Academy of Sciences under the grant no. IAA 100100627 also was enjoyed.

\section{References}

[1] W.M. McCall, K.S.V.L. Narasimhan, R.A. Butera, J. Appl. Crystallogr. 6, 301 (1973).

[2] H. Pinto, M. Melamud, E. Gurewitz, Acta Crystallogr. A 35, 533 (1979).

[3] P. Blaha, K. Schwarz, G. Madsen, D. Kvasnicka, J. Luitz, WIEN2k, TU Wien, Austria 2001.

[4] M. Diviš, J. Rusz, H. Michor, G. Hilscher, P. Blaha, K. Schwarz, J. Alloys Comp. 403, 29 (2005).

[5] W.L. Goffe, G.D. Ferrier, J. Rogers, J. Econometrics 60, 65 (1994). 